\title{
El Museo del Clima de Beniarrés (Alicante). Propuesta de un recurso didáctico para la enseñanza de la Climatología
}

\section{The Museum of the Climate of Beniarrés (Alicante). Proposal of a didactic resource for teaching Climatology}

\section{Álvaro Francisco Morote Seguido Enrique Moltó Mantero}

Instituto Interuniversitario de Geografía

Universidad de Alicante

\begin{abstract}
Resumen: Las visitas escolares a centros de interpretación se han convertido en los últimos años en uno de los mejores recursos didácticos para llevar a la práctica lo aprendido en el aula. El trabajo que aquí se presenta tiene como objetivo potenciar y ofrecer el Museo del Clima de Beniarrés (Alicante) como un recurso didáctico para la asignatura de Geografía (o afines) en la que se aborden aspectos relacionados con la Climatología y su relación con el medio y la sociedad con la explicación de ejemplos y experiencias, tanto por parte del alumnado como del profesorado. Este museo se convierte en uno de los primeros centros con finalidad didáctica de esta tipología en España. Como conclusión, cabe indicar las múltiples ventajas y alternativas que ofrece este museo y especialmente, conseguir que el alumnado aprenda "jugando".
\end{abstract}

Palabras clave: Didáctica; Geografía; Climatología; Museo del Clima de Beniarrés.

Abstract: In recent years, school visits to interpretation centers have become one of the best didactic resources to put into practice the things that it has been learned in classroom. The aim of this research is to promote and offer the Museum of the Climate of Beniarrés (Alicante) as a didactic resource for the subject of Geography (or related) in which aspects are related with Climatology and its relationship with the environment and society with the explanation of examples and experiences from the students and the teachers. This museum becomes one of the first centers with didactic purposes of this typology in Spain. As conclusion, it is worth mentioning the many advantages and alternatives that offer this center and especially to get students learning "playing".

Keywords: Didactics; Geography, Climatology, Museum of the Climate of Beniarrés.

(Fecha de recepción: febrero, 2017, y de aceptación: julio, 2017)

DOI: 10.7203/DCES.32.9624 


\section{Introducción}

La Geografía es la ciencia de las relaciones entre el hombre (la sociedad) y la naturaleza (el medio). Su concreción se manifiesta siempre en un espacio concreto (un territorio) cuya apariencia fisionómica da lugar a un paisaje que, a su vez, es una elaboración cultural (Martínez-Fernández et al., 2015). Una de las diversas ramas científicas de la Geografía es la Climatología, entendida ésta como la ciencia que se ocupa del estudio del clima y de sus variaciones a lo largo del tiempo. El estudio del clima constituye una tarea fundamental en la formación del alumnado tanto en la educación primaria como secundaria. En primer lugar, porque el currículum vigente así lo establece en un contenido a enseñar en todos los niveles educativos. Y en segundo lugar, debido a la enorme transcendencia social que tiene gran parte de las actividades en la vida cotidiana (Tonda y Sebastiá, 2003). El conocimiento del clima ha estado presente en los diversos planes educativos, casi siempre vinculado a la enseñanza en las Ciencias Sociales, especialmente en la etapa de Educación Primaria mientras que en la Educación Secundaria, su aprendizaje ha estado separado, por un lado, en Ciencias Sociales, donde aparecen los contenidos relacionados con los climas y los dominios bioclimáticos, mientras que las cuestiones sobre tiempo atmosférico se engloban en la enseñanza de las Ciencias de la Naturaleza (Martínez-Medina y López-Fernández, 2015).
La enseñanza y aprendizaje de la Climatología en sus aspectos más relevantes se ha efectuado en la formación geográfica y en la enseñanza universitaria a través de la formación teórica y a partir de conocimientos proporcionados desde la Geografía Física, así como por el aprendizaje de las técnicas y los recursos de representación gráfica propios de la Climatología como disciplina como son climogramas, gráficos y mapas del tiempo (Valbuena y Valverde, 2006). Algunos de los ejercicios prácticos para desarrollar en actividades vinculadas con el clima suele ser habitual elaborar y representar los datos climatológicos existentes en un territorio. Por ejemplo, se efectúan dichos trabajos a partir de los datos de pluviometría, temperatura y medidas de presión de un lugar y se elaboran gráficos y diagramas de la evolución del tiempo atmosférico, lo que permite conocer los rasgos climáticos más relevantes de un territorio.

Una de las ventajas que ofrece la ciencia geográfica es su capacidad de poder enseñar elementos del territorio fuera del aula. Diversos estudios indican que el uso del libro de texto y de la metodología expositiva clásica siguen siendo predominantes en el aula de secundaria de ciencias sociales (Martínez et al., 2009) y especialmente en la Geografía del Bachillerato (De Miguel, 2013), con los efectos que ello tiene, al convertirse en una asignatura ardua, extensa, compleja y, consecuentemente, poco atractiva para el alumnado. Frente a ello, existen diferentes aportaciones 
que incitan a desarrollar el concepto de clase creativa (Bocconi et al., 2012) que, en el caso de la Geografía, está condicionado esencialmente por los presupuestos constructivistas (Roberts, 2011). En este sentido, una forma de romper con las rutinas escolares es el uso de salidas de campo o itinerarios didácticos a áreas relacionadas con lo visto en clase, incluyendo áreas rurales, urbanas, centros de interpretación, etc. Ello permite que la Geografía se convierta en una materia más atractiva y en la que los alumnos se encuentren más motivados para el aprendizaje.

A finales del siglo XIX, fue la Institución Libre de Enseñanza la que adoptó las excursiones como parte fundamental de su nuevo enfoque pedagógico, ya que en ella se daban las mejores condiciones para lograr la educación completa e integradora del ser humano (Ortega, 1998: 81-113). En 1886, Francisco Giner de los Ríos y Manuel Bartolomé Cossío, realizaron la primera excursión pedagógica de la Institución a la Sierra madrileña, hecho que supondría una nueva puesta en valor del paisaje serrano, el denominado "guadarramismo". Sin embargo, hoy en día, la Geografía tradicional que se enseña a través de las prácticas escolares y la propia opinión pública se resiste a las propuestas de innovación que pretenden responder a las exigencias del contexto socio-ambiental para superar los nuevos desafíos ciudadanos (Souto, 2010). Como resultado, algunos conceptos que se aprenden en el aula como el espacio geográfico, espacio o parque natural difiere con la información que el alumnado recibe constantemente desde su propia realidad vivida (Santana et al., 2015).

El conocimiento fuera del aula a partir de salidas de campo o de trabajo supone una herramienta de primer orden para el descubrimiento, comprensión e interrelación de los elementos físicos que conforman el entorno que nos rodea. Su realización conlleva un aprendizaje en el que esos elementos y su papel en el escenario, sus relaciones, son entendidos como una unidad funcional (Crespo, 2012). Prueba del alto rendimiento que este tipo de recursos se puede obtener, son los numerosos itinerarios didácticos que en los últimos años han sido publicados en diferentes revistas y congresos relacionados con la didáctica de la Geografía (Mínguez, 2010). Es el caso, por ejemplo, de las visitas al Laboratorio de Climatología de la Universidad de Alicante que desarrolla actividades de divulgación y didáctica, no sólo en las asignaturas propias del Grado de Geografía y Ordenación del Territorio de la propia universidad, si no que se llevan a cabo visitas por un surtido número de colegios y centros de secundaria de la provincia de Alicante (Morote, 2015).

Numerosos autores han resaltado el indiscutible potencial didáctico de las salidas de campo y el aprendizaje fuera del aula en la enseñanza de las Ciencias Sociales en general y de la Geografía en particular (Sánchez, 1995; Marrón, $2001 ; 2011$ ), coincidiendo en que la visita al territorio ofrece al alumnado una 
posibilidad de comprensión y relación de los hechos geográficos difícilmente alcanzable por medio de otros recursos, facilitando el aprendizaje significativo a través de la experiencia de campo.

\section{Objetivos y Metodología}

El objetivo de esta contribución es proponer y potenciar las posibilidades y recursos didácticos que ofrece el Museo del Clima de Beniarrés (provincia de Alicante) como una herramienta para la enseñanza de la Climatología. Para ello, se han expuesto dichas potencialidades y ejemplos de actividades de cómo este centro puede servir para la enseñanza de la Climatología para los alumnos y como recurso didáctico disponible para el profesorado.

El centro de interpretación aquí propuesto se trata de un magnífico ejemplo para poder enseñar temas que se enseñan en las aulas en relación con la Climatología y su vinculación con el medio y la sociedad. Además, este centro ofrece numerosas formas de aprendizaje que el alumnado puede desarrollar durante su visita y complementar con lo que se ha dado en clase y que se puede proponer tanto en niveles escolares de primaria, secundaria e incluso universitarios. También cabe indicar que dada la situación geográfica en la que se encuentra (Montaña de Alicante), y en una localidad con una marcada actividad rural, ofrece una alternativa didáctica que se puede complementar con la realización de otras actividades por el término municipal de Beniarrés con el objetivo de ver en el paisaje lo visto en el museo.

Desde las perspectivas más actuales, la didáctica de la Geografía escolar se funda en el convencimiento de hacer partícipes a los niños en la construcción de su propio conocimiento y en la adquisición de las competencias básicas, a través de la interactuación con el entorno que los rodea y del que forman parte (Bale, 1996). Por lo tanto, el trabajo que aquí se presenta parte de la motivación de desarrollar un recurso útil y de verdadero valor para los maestros de Educación Primaria, Secundaria y/o universitarios que quieran innovar, aprender, complementar sus metodologías docentes y llevar a la práctica lo visto en clase.

Metodológicamente se llevaron a cabo diferentes entrevistas en noviembre de 2016 con los precursores de esta iniciativa didáctica (Luis Tomás -anterior alcalde de Beniarrés-, Enrique Moltó y Jorge Olcina -profesores del Departamento de Análisis Geográfico Regional y Geografía Física de la Universidad de Alicante-). Con la consecución de estas entrevistas se recogió información, tanto de los recursos didácticos que ofrece este centro como de diferentes ejemplos prácticos y experiencias didácticas de las visitas que se han llevado a cabo.

Durante la realización de las entrevistas, además, se realizó una visita guiada por el centro de interpretación para poder enseñar in situ los recursos didácticos que ofrece este museo al igual que una visita por los parajes más 
interesantes y "potencialmente didácticos" del término municipal de Beniarrés que pueden complementar la visita al Museo del Clima.

El cuestionario de la entrevista se estructuró en los siguientes apartados:

1. Iniciativa de la propuesta

2. Objetivos y finalidad

3. Dificultades a la hora de poner en marcha el centro

4. Ubicación y propiedad del centro

5. Personal, horarios y visitas guiadas

6. Destinatarios potenciales

7. Herramientas y recursos didácticos del centro

8. Actividades que se llevan a cabo (talleres, seminarios, jornadas, etc.)

9. Originalidad y potencial didáctico de este centro para la enseñanza de la Climatología

10. Actividades y estrategias de difusión del centro

11. Posibilidad de integrar la visita del centro con el entorno de la localidad de Beniarrés

12. Debilidades y propuestas de mejora

\section{El Museo del Clima de Beniarrés. Estudio de caso}

La puesta en marcha del museo: Iniciativa, finalidad, dificultades $y$ difusión

El Museo del Clima de Beniarrés, aunque gira esencialmente en torno al peculiar clima de la Montaña de Alicante (comarcas de l'Alcoià, El Comtat y partes de l'Alacantí y Las Marinas), es realmente un centro de interpretación donde el visitante puede conocer las características geográficas de las comarcas del interior de Alicante. Aprovechando las peculiaridades de este clima y su relación con hechos y fenómenos físicos $\mathrm{y}$, sobre todo, geográficos (tanto físicos como humanos), se explican fenómenos que perfectamente se pueden exportar a otros territorios. El principal objetivo del Museo es, por tanto, explicar y dar a conocer algo tan cercano y cotidiano como son los condicionantes geográficos (esencialmente climáticos), que dan personalidad al paisaje y que explican la forma de vida de la sociedad del territorio circundante.

El Museo del Clima se localiza en la población de Beniarrés, un pequeño pueblo de tan sólo 1.500 habitantes situado en el norte del interior de la provincia de Alicante (Comarca de $E l$ Comtat) (Figura I). El centro se ubica en la antigua Casa del Maestro (Calle San Vicente, 10). En este sentido, cabe hacer notar que tradicionalmente, en estas pequeñas localidades era habitual que el ayuntamiento cediera una vivienda para que residiera el maestro durante el curso. Y, a día de hoy, no deja de ser curioso que la función didáctica del museo conecte con esta función tradicional. Tanto el edificio como el Museo son propiedad del Ayuntamiento de Beniarrés, aunque la inversión, como es habitual en estas localidades rurales corre a cargo de la Diputación de Ali- 


\section{Figura I \\ Panorámica de la localidad de Beniarrés (provincia de Alicante)}

Fuente: http://beniarres.org/

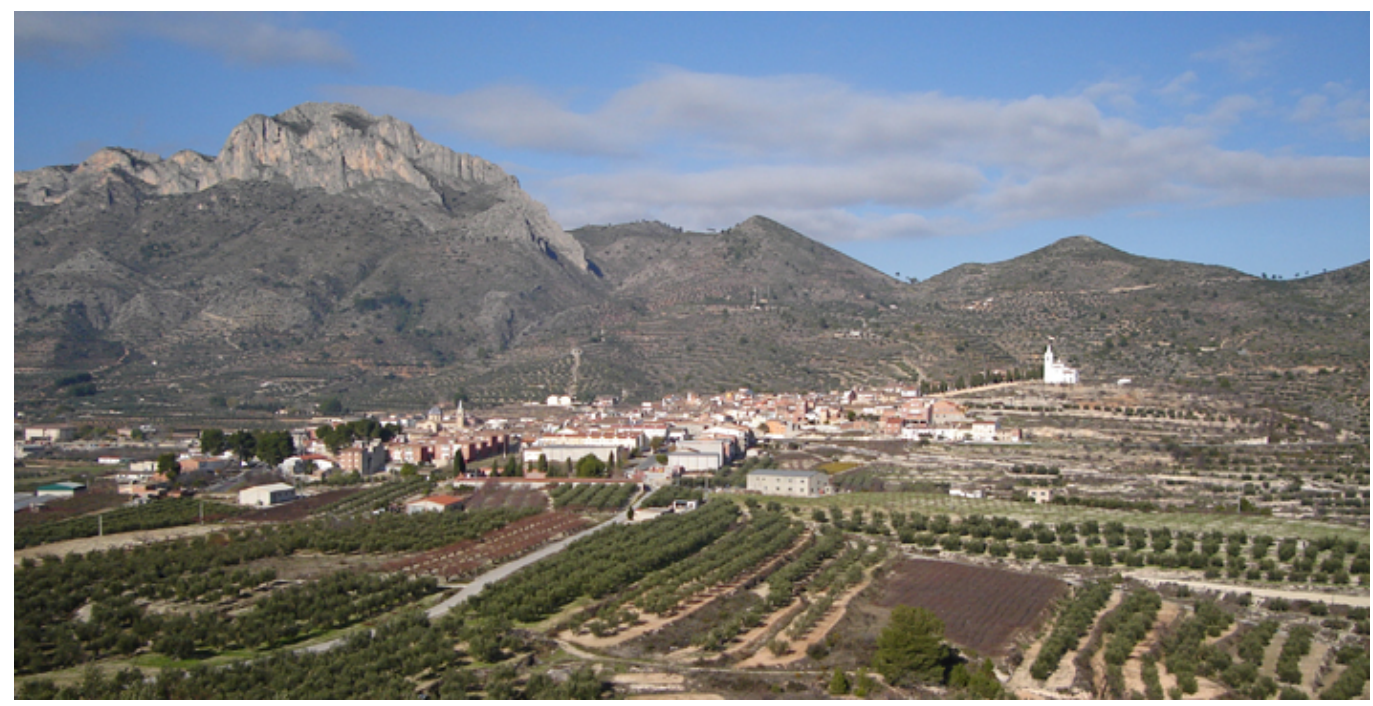

cante, de quien dependen también los fondos para costear a la persona encargada de abrir y cerrar el centro.

La iniciativa de poner en marcha el museo fue ideada en 2009 por el alcalde de la localidad aprovechando el espacio libre de un edificio que alberga un pequeño Museo Arqueológico en torno al yacimiento de la Cova de l'Or (Beniarrés) donde se muestran restos (especialmente cerámicas) del Neolítico Valenciano (5.600 a.C.). Dicha idea fue desarrollada con la colaboración de los profesores del Departamento de Análisis Geográfico Regional y Geografía Física de la Universidad de Alicante (Jorge Olcina y Enrique Moltó) que han desarrollado sus líneas de investigación en torno a la Climatología, los cuales, propusieron al alcalde ideas y propuestas didácticas e intentando que se desarrollara un centro relacionado con el clima de la Montaña de Alicante. Finalmente, el 26 de marzo de 2011 se inauguró el museo coincidiendo con la celebración de unas jornadas de aficionados a la Meteorología y la Climatología ("Els aficionats profesionalitzats de la Meteorologia i la Climatologia"), fundamentales para este centro y que se siguen celebrando anualmente hasta la actualidad.

De forma guiada, el museo se puede visitar únicamente los fines de semana, los sábados en horario de mañana (10:30-14:00) y tarde (18:30-21:00) y los domingos por la mañana (10:30-14:00). En esos días y horario, hay una persona 
encargada de abrir y cerrar el centro y que, además, da la casualidad que por su formación (Máster en Gestión del Patrimonio) ayuda a realizar las tareas de guía. No obstante, en su orientación didáctica, lo más interesante es ofrecer las visitas del centro durante los días lectivos que es realmente cuando los escolares pueden visitarlo. En este sentido, si hay una visita escolar, el responsable se pone en contacto con el ayuntamiento y un funcionario abre el edificio en un horario acordado, eso sí, sin visita guiada. Por ello, sería deseable que los responsables o profesores de los grupos de alumnos fueran conocedores de las materias que se ofrecen en el Museo y, a ser posible, que recibieran algún tipo de material o formación específica de los contenidos del mismo.

Por ello, sería necesaria la elaboración de una guía didáctica para ser repartida entre los docentes de los colegios que piensen impartir e incluso, unos cursos para docentes del Cefire (Sevicio de Formación del Profesorado de la Conselleria de Educació, Cultura $i$ Esport de la Generalitat Valenciana que se encarga mediante cursos y otras actividades de mantener una formación continua para todos los docentes de la Comunidad Valenciana), por ejemplo, con visitas al propio museo. A partir de aquí convendría que se planteará una guía docente en los colegios de la comarca de El Comtat (y que ésta fuera común), distinta en función del curso y siempre vinculada a conocimientos geográficos más generales aplicados al entorno cercano. Con ello, el profesor podría hacer la visita guiada con unos conocimientos previos que pueden complementar, llevar a la práctica y enseñar en el museo lo visto en clase. Además, una limitación a tener en cuenta en la función didáctica de este centro es la capacidad de carga física ya que, lo idóneo, sería que los grupos se distribuyesen entre 10 y 15 alumnos por planta, por lo que las visitas han de ser debidamente planificadas en calendario y horario.

Una de las grandes dificultades a la hora de montar este centro fue la disponibilidad en propiedad de instrumentos de medición de elementos del clima que, a modo de exposición, servirían para mostrar de primera mano a los visitantes cuáles han sido y son los diferentes aparatos que se usan para la recolección de datos climatológicos. En este sentido, según las opiniones mostradas por los precursores, "la parte instrumental siempre ha sido un problema porque nadie ha cedido aparatos meteorológicos más o menos antiguos (termómetros, pluviómetros, barómetros, barógrafos, termógrafos, pluviógrafos, etc.). Por ello, hasta el momento, en relación con el aparataje climatológico sólo se cuenta en propiedad con una estación meteorológica automática y que, además, forma parte también del proyecto museístico. Ante este contratiempo, cabe advertir que determinados centros públicos (Instituto Interuniversitario de Geografía de la Universidad de Alicante) o incluso, particulares, en determinadas ocasiones, han cedido sus instrumentos de forma temporal coincidiendo con 
algún evento o jornada celebrada en el Museo.

En relación con las tareas de difusión y divulgación, básicamente dependen de los contactos con los medios de comunicación de los precursores del centro, sobre todo con los encuentros de aficionados que suelen atraer a más de un centenar de personas por los invitados destacados que vienen. Además, en la emisora decana de la Comunidad Valenciana, "Radio Alcoy" de la Cadena Ser son numerosas las referencias al Museo del Clima en el programa dedicado a la "Meteorología y Climatología" que, con carácter diario, realiza el profesor Enrique Moltó. Destaca una entrevista en el programa de radio "No es un día cualquiera" de Pepa Fernández con el responsable de la página web "Divulgameteo" y su hombre del tiempo Jose Miguel Viñas que también suele citar el museo en alguna que otra ocasión. Además, cabe indicar la labor de divulgación del museo que el propio profesor Enrique Moltó realiza continuamente por los colegios de la comarca para hablar sobre el clima y su vinculación con la sociedad de la Montaña de Alicante.

\section{La potencialidad didáctica del museo:} Recursos, experiencias y originalidad para la enseñanza de la Climatología

El Museo del Clima de Beniarrés, aunque su finalidad principal es la didáctica de la Climatología, como su propio nombre indica también se convierte en un centro de exposición que puede ser visitado para todos los públicos. El material ubicado en la planta baja está orientado a niños pequeños (primeros años de primaria) donde se encuentran mapas de España y símbolos magnéticos para que ellos mismos jueguen y elaboren sus mapas de tiempo como los que se pueden observar en los programas de televisión sobre el tiempo (Figura II). La planta baja, por tanto, sirve de introducción. No conviene olvidar que también en esta parte hay una sala preexistente al Museo del Clima de Beniarrés que explica el yacimiento de la Cova de l'Or, que es perfectamente compatible con la visita didáctica en torno al clima.

En la primera planta, la mayoría de los paneles explicativos pueden ser entendidos por gran parte de los visitantes con una cultura media en ciencias naturales y/o sociales. Se dispone de un video y un 3D que pueden ser un buen resumen de lo explicado en los paneles, a la vez que, debidamente planteados, puede convertirse en una forma de poner de manifiesto que los conocimientos han sido asimilados. Estos paneles pueden estar especialmente orientados para alumnos de algunas asignaturas de secundaria e incluso, de algunos grados universitarios que tengan alguna relación con el clima (Geografía, Turismo, Ciencias Ambientales, etc.). De hecho, desde su funcionamiento en marzo de 2011 el museo ha sido visitado por alumnos de $4^{\circ}$ de primaria y de $4^{\circ}$ de ESO de diversos centros de la comarca, de alumnos del Grado de Geografía y Ordenación del Territorio de la 


\section{Figura II}

Mapas de tiempo con símbolos magnéticos para que interactúe el alumnado

Fuente: Foto de los autores.

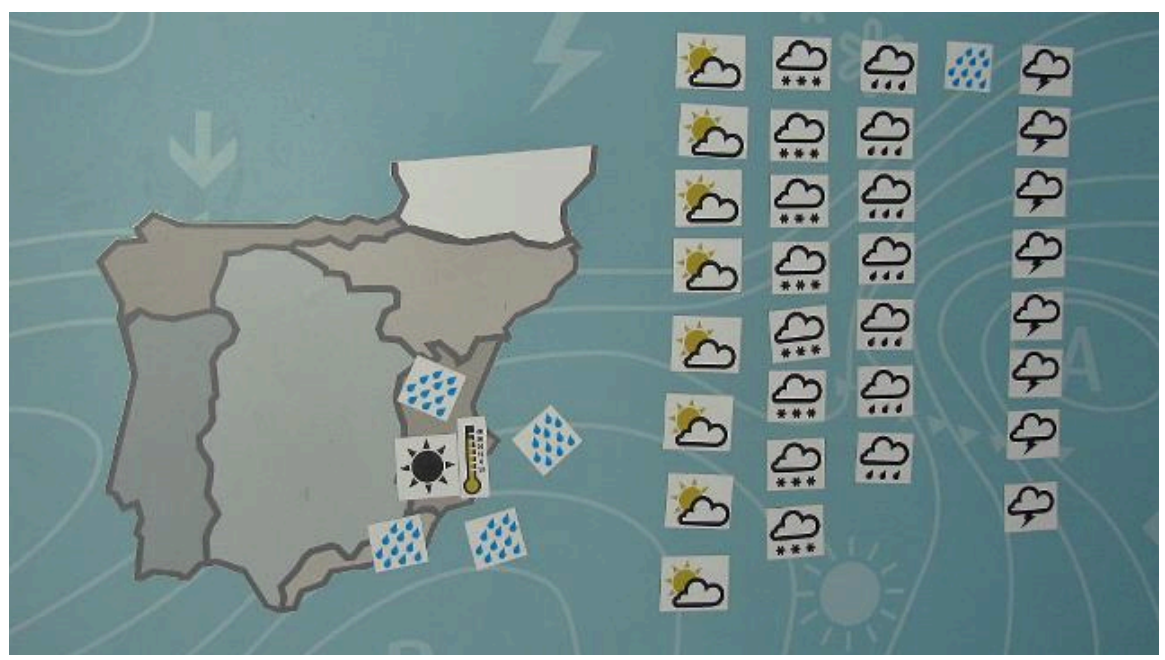

\section{Figura III}

Paneles didácticos con descripción de la Geografía y el paisaje de la Montaña de Alicante

Fuente: Foto de los autores.

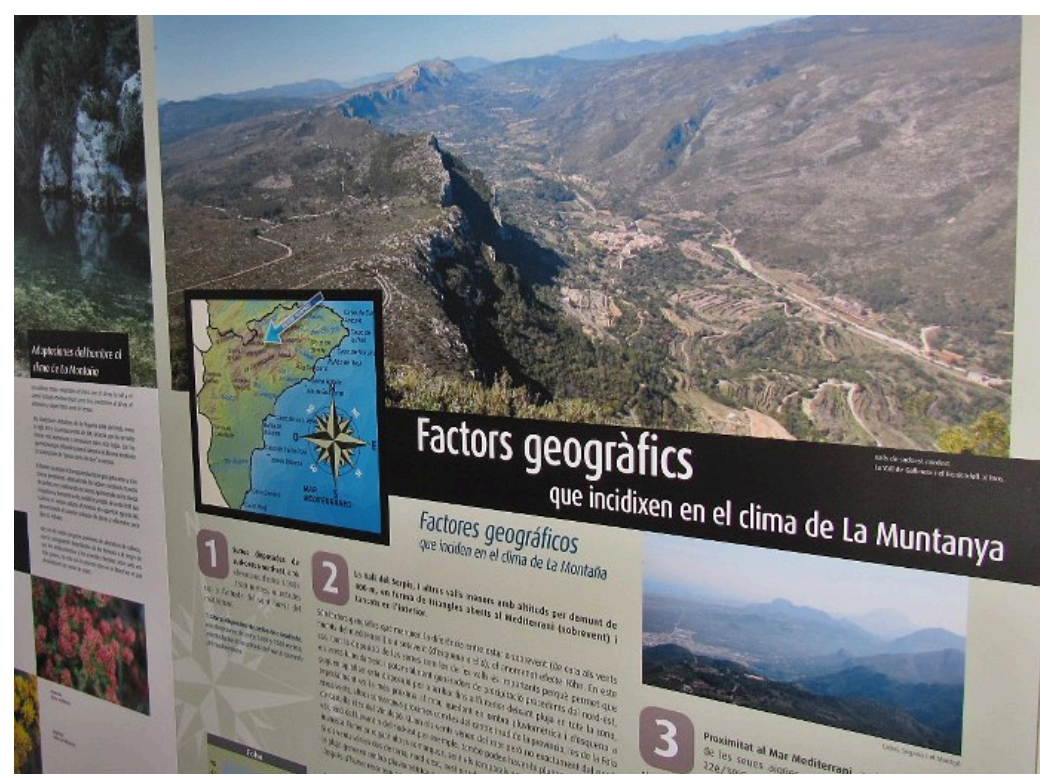


Universidad de Murcia y de la Universidad de Alicante (Grado de Geografía y Ordenación del Territorio, Máster de Planificación y Gestión de Riesgos Naturales y Universidad Permanente para adultos).
En el acceso derecho de la primera planta hay paneles con descripción básica de los conceptos de Meteorología y Climatología y un video con los principales desastres climáticos del mundo. En el acceso izquierdo se ubican dife-

\section{Cuadro I \\ Tradiciones de alto valor etnográfico pero con poco valor científico expuestos en el Museo}

Fuente: Elaboración propia.

> Las cabañuelas. Practicadas en toda España y de pretendida validez sólo para territorios reducidos, intentan pronosticar el tiempo de todos los meses del año. Los más atrevidos se aventuran incluso con hacerlo día por día, a partir de la observación de las condiciones de viento y humedad presentes a lo largo de 24 días de agosto, dando lugar a las cabañuelas de ida del 1 al 12, y a las de vuelta, del 13 al 24 .

> Según como amanezca el día 15 de agosto (festividad de la Virgen), así será el año. Si hay nubes o rocío abundante se supone que el año siguiente será más lluvioso que si el día amanece más seco.

$>$ Los calendarios de cebolla. En este caso, en la mágica noche de San Juan, se parten doce trozos de cebolla y se coloca encima de cada uno un poco de sal. Junto a cada trozo se coloca el nombre de un mes del año. A la mañana siguiente, si la sal ha sido más o menos absorbida significa que será un mes lluvioso y si no, será un mes seco.

$>$ La posición de la luna. Esta tiene un alcance de previsión más corto, el mes lunar. Si la luna cuando empieza a dibujarse como creciente presenta cierta verticalidad se dice que está "marinera" y en ese caso el mes lunar será lluvioso. Si, por el contrario, está en posición horizontal es preludio de mes seco.

$>$ "La luna de octubre hasta siete cubre". Se supone que si en el mes lunar de octubre hay lluvias es un indicador de que estas van a durar al menos siete meses lunares más.

$>$ Existe la creencia entre algunos agricultores de que si hay heladas la noche del 24 al 25 de diciembre es una señal de que la cosecha de almendras cuando llegue el momento se acabará helando. 
rentes paneles con una descripción de la Geografía y el paisaje de la comarca de El Comtat como muestra de su relación natural y antrópica con el clima (Figura III). En una sala intermedia, bastante amplia, los paneles muestran especial atención a los usos y dichos relacionados con el clima, especialmente asentados en la comarca, situándose en un lado, aquéllos con nula base científica pero can gran valor patrimonial (Cuadro I) y en otro, algún refrán y prácticas que suman al valor patrimonial cierto valor científico basado en la observación empírica (Cuadro II). También hay una pantalla que muestran diversos paisajes de la Vall de Perputxent (valle articulado en torno al río Serpis e integrado por las localidades de Alcocer de Planes, Gaianes, Beniarrés y L’Orxa).

En la "Sala Grande" y última, en el orden de visita, se encuentran tres puntos didácticos básicos:

1. Pantalla con explicaciones en calidad 3D de los fenómenos climáticos más destacados.

2. Video con explicación en mapas y paisajes de las causas y consecuencias del clima.

3. Paneles con explicaciones de los vientos y los principales centros de acción que afectan a la Península Ibérica (Figura IV).

También cabe indicar que los precursores del centro pusieron de manifiesto que una buena opción, aparte de visitar el propio museo, es llevar a cabo una jornada completa de salida de campo por los alrededores del municipio como actividad complementaria a lo visto en el centro. En este sentido, cabe señalar que existen rutas señalizadas como tal por todo el término municipal de Beniarrés que se podrían potenciar. Parecen especialmente vinculadas y que suelen hacerse a la Albufera de Gaianes, al Pantano de Beniarrés, al Barranc de la Encantà y al yacimiento arqueológico neolítico de la Cova de l'Or, etc.

\section{Figura IV}

\section{Panel didáctico de los principales tipos y dirección de viento y centros de acción que afectan a la Península Ibérica}

Fuente: Foto de los autores.

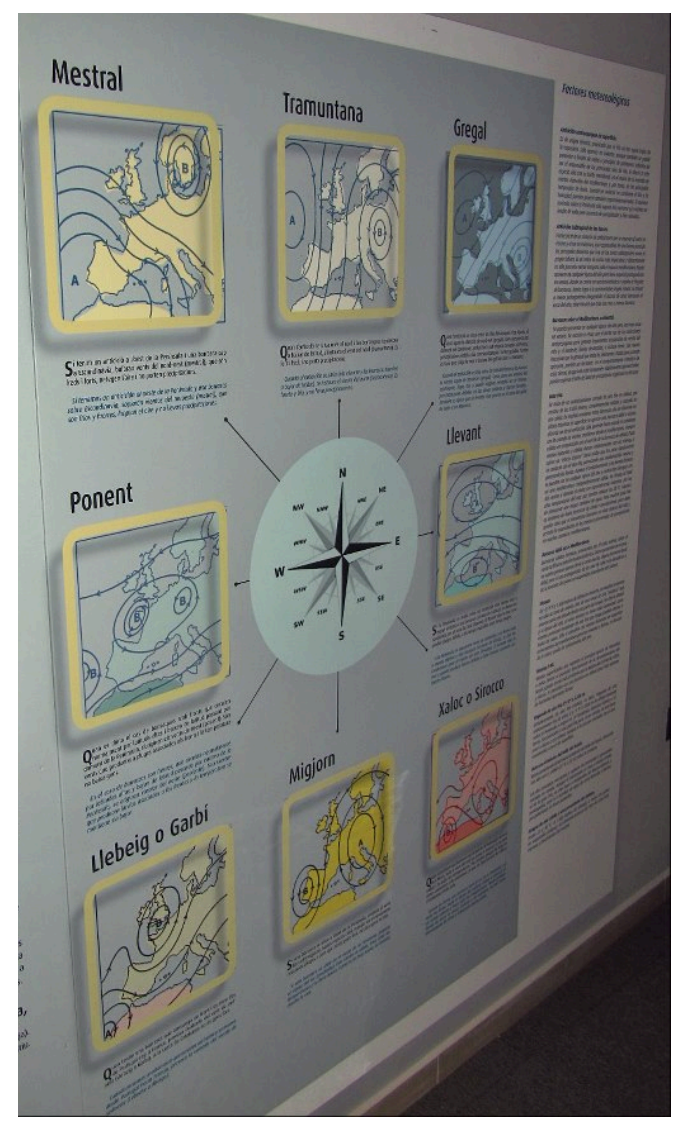




\section{Cuadro II \\ Refranes y observaciones locales con cierta base empírica expuestos en el Museo}

Fuente: Elaboración propia.

-Boira en Benicadell, pica espart y fes cordell”. Refrán que hace referencia precisamente a la sierra próxima que se sitúa a la entrada de los vientos húmedos del nordeste para toda la comarca y por tanto, es la puerta de entrada de los temporales de lluvia. La idea central es que cuando llovía y no se podía trabajar en el campo los agricultores se dedicaban a otras labores necesarias como la de hacer utensilios o alpargatas de esparto. Es decir, con la aparición de esta niebla en el Benicadell, significaba un precursor de un temporal de lluvias y el agricultor tenía que dedicarse a estos labores preparando el esparto para su manipulación.

$>$ "Pel llebeig aigua veig". En este caso se hace referencia a que este viento del sudoeste puede traer algunas lluvias. Cuando las borrascas vienen del oeste no suelen traer apenas precipitación pero si proceden del sudoeste es porque tienen algo más de profundidad y sí pueden dar lugar a algunas lluvias moderadas (siempre menos importantes que los temporales de origen mediterráneo). De todas formas este refrán está más acertado si se completa con el que reza "el llebeig la mou i el llevant la plou". En este caso, es una auténtica lección de Climatología Aplicada ya que efectivamente las borrascas que entran por el Golfo de Cádiz a veces entran primero con viento del sudoeste ("el llebeig la mou") y luego, por el giro ciclónico en contra de las agujas del reloj, los vientos acaban rolando a marítimos, mucho más húmedos, y es cuando se da un temporal de lluvias importantes ("el llevant la plou").

$>$ "Vent de Tramuntana no te abric i home pobre no te amic". En este caso se habla del viento más frío que afecta a este territorio, Tramuntana o norte, que era muy difícil de combatir con los medios de otros tiempos. Hay más refranes que hacen referencia a un viento que está ligeramente inclinado hacia el este, entre la Tramuntana y el Llevant. Se trata del Gregal o nordeste, protagonista de los principales temporales de lluvia y que recibe la ilustrativa denominación de "plovença", al introducirse a través del Valle del Serpis desde el mar sin perder tanta fuerza hacia el interior como lo hace cuando viene desde el este, ya que, en este último caso, tropieza antes con las montañas y las lluvias quedan más concentradas en el litoral y prelitoral de la Marina Alta (provincia de Alicante) y la Safor (provincia de Valencia). 
"Cel a borreguets pluja o neu a cabassets". Los altocúmulos son nubes de tipo medio y alto pequeñas y numerosas que dan esa sensación de un cielo lleno de "algodones". Cuando estas nubes aparecen por el este y, sobre todo, por el nordeste son el aviso inequívoco de la llegada de una frente en las próximas 24 a 36 horas porque son los segundos en aparecer, después de las pinceladas de los cirros y antes que las nubes que traerán la precipitación de lluvia o nieve y por tanto tienen una base "científica" indiscutible. En este punto conviene advertir que en primavera y verano, cuando la atmósfera está un tanto inestable y puede dar lugar a la aparición de nubosidad de evolución y de tormentas vespertinas, aunque el cielo amanezca completamente despejado, unas pocas nubes parecidas en forma a las que se han citado anteriormente encima de alguna de las sierras, los "altócumulus castellatus", son para muchos agricultores señal inequívoca de que por la tarde va a haber tormentas y no suelen equivocarse, aunque es más difícil saber cuánto y donde va a llover exactamente.

$>$ También es tradicional la observación de que si el día amanece con rocío abundante es difícil que ese día haya algún tipo de precipitación. Es un hecho que resulta lógico ya que el rocío es síntoma de estabilidad y situación anticiclónica, aunque, por supuesto, tiene un margen de error.

$>$ “Cel rogenc pluja o vent". Lo cierto es que en este territorio la aparición en la puesta de sol, hacia poniente, de un fondo nuboso de intenso color rojo es señal inequívoca de viento moderado a fuerte de poniente y en ningún caso de lluvia.

$>$ "Tindre rogle (halo) la lluna". La aparición de uno o dos pequeños arcoíris ("parasols") a los dos lados del sol cuando éste se va a poner es un indicador de cambio de tiempo como poco y, en general, de temporal de lluvias en las próximas 24 a 72 horas, ya que es reflejo de la aparición de cirros, nubes altas que dejan ver el sol e incluso la luna y que incluso descomponen los cristales de hielo en colores, y que son las primeras nubes en aparecer cuando se acerca un frente nuboso, y si eso coincide con vientos marítimos, querrá decir que vienen lluvias porque el frente procederá del mediterráneo.

$>$ "Arc de sant Martí pel matí, aigua esta ací, arc de Sant Martí per la vesprada aigua pasada". Dado que el arcoíris se forma cuando coinciden el sol y la precipitación, si aparece por la mañana lo vemos a poniente y quiere decir que el frente nuboso mediterráneo aún está llegando. Si por el contrario, aparece por la tarde, lo hará en el levante y querrá decir que el frente nuboso mediterráneo ya está a punto de pasar por completo. 
Como intentos de adivinar el tiempo a largo plazo pero con cierta base estadística y por supuesto, falible, se cree que por ejemplo, si "la Candelaria riu l'hivern es viu i si plora l'hivern és fora”. Es decir, se supone que, si llegado este día, el 2 de febrero, el invierno se muestra fuerte es síntoma de que está a punto de acabar mientras que si da síntomas de "debilidad" quiere decir que aún puede volver atrás y recrudecerse. También se supone que si el mes de marzo se parece al mes de mayo por "estadística" habrá una "vuelta atrás" y el mes de mayo se puede parecer al de marzo ("cuando marzo mayea, mayo marcea").

\section{Cuadro III}

Experiencias y ejemplos prácticos de actividades que se realizan

Fuente: Elaboración propia.

\section{Nivel Ejemplo de actividades}

Primaria -Explicación del mecanismo de la precipitación mediante una olla llena de agua que se calienta para explicar la evaporación y la formación de las nubes de vapor de agua y a la que se le coloca la tapadera encima en un momento dado. Con ello, los niños entiendan el mecanismo de la condensación al comprobar como en esa tapadera se vuelven a formar gotas liquidas como sucede en la atmósfera al encontrar una capa de aire frío en altitud.

-Pizarra de símbolos magnéticos. La actividad consiste en preguntar a los escolares qué efectos climáticos pueden suceder en relación con diferentes situaciones de tiempo. Para ello, los alumnos interactúan por grupos a modo de "juego".

-Información sobre dichos populares. La actividad consiste en preguntar a los escolares que situación de tiempo está asociada a los diferentes dichos relacionados con el clima. Por grupos, los alumnos tienen que intentar asociar y explicar el porqué de estas situaciones y por qué estos dichos se dicen.

-Recolección de datos. Esta actividad se propone como un ejercicio de "juego" donde alumno aprende a cambiar las bandas de determinados aparatos (pluviógrafos, barógrafos y termohigrógrafos, estación automática, etc.), para de esta manera comprender la importancia 
del almacenamiento de dicha información y observación de datos climáticos.

-Realización de climogramas. Gracias a la recolección de datos (temperatura y precipitación), se propone a los escolares (de forma individual) que realicen un climograma (anual o mensual) para que pueden comprender las principales características del tiempo de la montaña de Alicante.

-Realización de una roseta de los vientos. Con esta actividad, gracias a la recolección de datos de dirección de viento (estación automática), se propone a los alumnos (de forma individual) la realización de esta roseta de los vientos.

Secundaria -Información sobre dichos populares. La actividad consiste en preguntar a los escolares que situación de tiempo está asociada a los diferentes dichos relacionados con el clima. Por grupos, los alumnos tienen que intentar asociar y explicar el porqué de estas situaciones y por qué estos refranes se dicen.

-Recolección de datos. Esta actividad tiene el objetivo de que el alumno conozca cómo se lleva a cabo la recolección y almacenamiento de datos y cómo esta información puede ser de importancia para llevar a cabo estudios climáticos y comprender el comportamiento del clima. -Realización de climogramas. Gracias a la recolección de datos (temperatura y precipitación), se propone a los escolares (de forma individual) que realicen un climograma (anual, mensual o diario) para que pueden comprender las principales características del tiempo de la montaña de Alicante.

-Panel didáctico de los principales tipos y dirección de viento y centros de acción que afectan a la Península Ibérica. Esta actividad consiste en preguntar qué tipos de tiempo se asocian con los diferentes centros de acción y vientos dominantes que afectan a la Península Ibérica.

-Explicación mediante el uso de recipientes llenos de distintos tipos de suelos de cómo funciona la infiltración de la lluvia en el suelo en función de las distintas características de los materiales y de la presencia o no de cubierta vegetal. Es fundamental para que entiendan el funcionamiento de los acuíferos.

-Realización de mapas topográficos de la Sierra del Benicadell (la más cercana al Museo), Aitana (la más alta de la provincia de Ali- 
cante), El Menetjador y la Sierra de Mariola (con su papel de barrera climática entre las caras a umbría y barlovento de las lluvias y las de solana y sotavento de las mismas).

Universidad -Se plantea una visita desde el campus de San Vicente del Raspeig (Universidad de Alicante) para los alumnos de Climatología de $2^{\circ}$ del Grado de Geografía y Ordenación del Territorio y para los alumnos del Máster de Planificación y Gestión de Riesgos Naturales (también se puede enfocar para cursos de secundaria). En el primer caso se incide en el cambio paisajístico que se da entre el punto de origen (mediterráneo semiárido), el sector intermedio de la Hoya de Castalla (mediterráneo continentalizado) y los valles del Serpis (mediterráneo húmedo-subhúmedo). La idea es que perciban que en el contexto de un clima común, el mediterráneo, las diferencias de precipitación y temperatura dan lugar a muy diversos paisajes naturales y agrarios. Todo esto se puede percibir en apenas 70 kilómetros de distancia y el relieve con su función de altitud, orientación (umbría/solana) y exposición (barlovento/sotavento respecto a los vientos húmedos). También en este caso, pero más para los estudiantes del Máster, se incide en las causas y las consecuencias de los fenómenos naturales extremos de la comarca, susceptibles de convertirse en riesgos. Todo ello se ve durante la salida de campo, se explica en el Museo y se vuelve a ver en el viaje de vuelta

-Resulta útil la visita al entorno próximo del Museo en distintos lugares que no están a más de 15 minutos en autobús: El pantano de Beniarrés, para que entiendan cómo el hombre intenta aprovechar toda la irregularidad de la precipitación acumulando agua de periodos lluviosos para regar en periodos secos; el Barranc de la Encantà (entre Beniarrés y Planes) para que vean cómo funciona la regulación kárstica que hace de forma natural lo mismo que el pantano, almacenar lluvia bajo tierra fruto de periodos lluviosos para soltarla durante el resto del año. Lo importante es que entiendan este proceso, el abastecimiento a las poblaciones y que gracias a la regulación karstica se evite por tanto las importantes evaporaciones que se dan en este clima; la Albufera de Gaianes explica muy bien cómo funciona un área endorreica y su relación con la irregularidad de las lluvias, que llena y vacía este espacio, y ejemplifica el gran valor de estas zonas húmedas en lugares secos a modo de oasis para vegetación y avifauna. 
-También se plantea para los futuros geógrafos, y para los estudiantes de turismo, la función del Museo como potencial recurso turístico y como modelo de proyecto a desarrollar por ellos mismos en otros contextos. La evaluación se puede hacer mediante una memoria del conjunto de la salida pero previamente es preferible realizar un cuestionario breve en clase sobre todo lo explicado, con preguntas que el alumno puede tener a su disposición antes de la salida, para saber en qué fijarse. Durante la visita se puede fomentar la participación con preguntas directas.

En relación con las metodologías de aprendizaje que el profesorado puede adquirir, gracias a este museo, los maestros cuentan con un lugar donde, gracias a los instrumentos de medición, paneles y elementos audiovisuales interactivos se pueden entender integradamente las interacciones entre el clima y los elementos físicos y geográficos que hacen que, de alguna forma, toda sea causa y consecuencia dando como resultado un paisaje agroforestal y una forma de vida de la población. La preparación de una guía didáctica por escrito, específica para cada nivel, la impartición de cursos para docentes, guías turísticos y monitores ambientales sobre los contenidos del museo y el propio aprendizaje del docente, cuando el autor de contenidos del museo se explica, tanto en el propio museo como en las visitas a los centros escolares son imprescindibles para que las visitas al Museo cobren sentido sin necesidad de que dicho autor tenga que explicar siempre en persona todos esos contenidos.
A parte de la labor museística y didáctica que ofrece este centro, el museo también se convierte en un lugar donde se celebran reuniones científicas, seminarios, exposiciones, etc. Un ejemplo de ello fue la realización de una exposición fotográfica de nubes montada por la asociación de aficionados "Cazatormentas" que estuvo expuesta entre noviembre de 2013 y marzo de 2014, gracias a Empar Landete. Además, todos los años (desde 2011) se celebra un encuentro de aficionados a la Climatología y la Meteorología, con entrada totalmente libre, que tiene una estructura en la que se invitan a varios ponentes tanto técnicos (Javier Miró, César Azorín, Jorge Olcina, María José Estrela, en los pasados años) como a nivel mediático (Manuel Toharia, Jordi Payá, Joan Carles Fortea, Victoria Rosselló y Jacob Petrus). En lo que se refiere a la vertiente más didáctica, también cabe hacer notar que en 2014 se celebró un taller de montajes de aparatos meteorológicos caseros (organizado por Jordi Payá). 


\section{Discusión y conclusiones}

Como ha sido enfatizado por reputados pedagogos, caso de María Montessori, "para enseñar y lograr aprendizajes significativos, nada mejor que el contacto directo con el entorno..." (Cordero, 2005: 95). Por lo tanto, el itinerario guiado se convierte en un excelente método para la enseñanza y el aprendizaje de la Geografía: la observación directa sobre el terreno de las formas y procesos espaciales (MartínezFernández et al., 2015).

El estudio del clima constituye una tarea fundamental en la formación del alumnado (en todos los niveles) debido a la enorme transcendencia social que tiene para gran parte de las actividades de la vida cotidiana. La asignatura de Geografía (o afines) permite numerosas actividades fuera del aula que se pueden utilizar como activadores de aprendizaje: actividades de orientación con planos, análisis de espacios urbanos, indagación sobre problemas ambientales, estudios de paisaje, actividades geológicas, etc. Trasladar los alumnos del aula no garantiza por sí solo mejores aprendizajes, ni siquiera mayor motivación. Para que esto se cumpla, la actividad fuera del aula tiene que estar integrada con lo que se trabaja en clase, que los alumnos tengan que hacer algún trabajo durante la jornada o incluso, que la actividad sea evaluada. Para conseguir que la salida de campo cumpla el objetivo enseñar y poner en la práctica lo que se ha visto en clase hay que procurar motivar al alumno.
Lo más importante es que éste quiera aprender, que tenga una predisposición positiva ante las tareas escolares, que conecte con la cultura escolar, que piense o sienta que eso es importante y que le va a ser útil para su vida futura y cotidiana. En definitiva, como indica Sandoya (2005), que esté motivado por aprender.

La visita al espacio geográfico puede, y debe considerarse, la mejor herramienta para interpretar, conocer y valorar un territorio. Sólo a través del análisis vivencial se puede comprender de manera significativa el papel que desempeñan las partes en el conjunto, es decir, ser capaces de identificar las relaciones que entre todos los elementos configuran la realidad que contemplamos. García (1994) considera que con los trabajos de campo, la Geografía se convierte en la mejor disciplina que pone en contacto al alumno con la realidad espacial. Numerosos autores han resaltado el indiscutible potencial didáctico de las salidas de campo en la enseñanza de las Ciencias Sociales en general y de la Geografía en particular (Sánchez, 1995; Marrón, 2001), coincidiendo en que la visita al territorio ofrece al alumnado una posibilidad de comprensión y relación de los hechos geográficos difícilmente alcanzable por medio de otros recursos, facilitando el aprendizaje significativo a través de la experiencia de campo.

Por ello, las salidas de campo sobre la interpretación del espacio geográfico se convierte en un recurso didáctico de primer orden (Martínez-Fernández et 
al., 2015). Numerosos ejemplos muestran como este recurso didáctico puede servir para interpretar el territorio. Por ejemplo Crespo (2012) describe la potencialidad de un itinerario didáctico para interpretar los elementos físicos de los paisajes de la Sierra de Guadarrama o en el caso colombiano con la interpretación del medio urbano y sus implicaciones didácticas (González y García, 2014). A través de las salidas de campo se pueden abordar conceptos, procedimientos y actitudes de tal modo que, apoyados en los trabajos de síntesis en el aula, proporcionen un aprendizaje significativo, sin que su puesta en práctica requiera más tiempo que el desarrollo de otras actividades ceñidas al centro. Por todo, la visita al Museo del Clima de Beniarrés (como otras salidas de campo), puede ser el mejor punto de partida para alcanzar la comprensión de los hechos geográficos en el proceso enseñanza-aprendizaje.

Las potencialidades didácticas que ofrece el Museo del Clima de Beniarrés son múltiples (Figura V). Una de ellas es que este centro permite conocer de primera mano y llevar a la práctica lo que se enseña en clase, especialmente, para los escolares de primaria en la que se empiezan a introducir temas relacionados con las ciencias naturales y sociales. También, porque este centro permite aprender "jugando" como pusieron de manifiesto sus precursores con la existencia de simuladores de tiempo mediante la introducción de diferentes elementos del clima y como los condicionantes del relieve pueden resultar determinantes en la manifestación de los tipos de tiempo y fenómenos extremos. Además, el aprendizaje que ofrece este centro, no sólo se enmarca en la Climatología, si no que, lo realmente importante y didáctico es la relación existente entre esta ciencia y su vinculación y manifestación en la sociedad y el territorio de la Montaña de Alicante. Por lo tanto, una manera interactiva de no perder la "Cultura del Territorio", especialmente en las edades más jóvenes y sobre todo, teniendo en cuenta las características socio-económicas de la comarca donde se ubica el museo (una comarca de interior) donde la actividad principal es la agricultura (pero cada vez más a tiempo parcial) y destacando, la pérdida de población joven que, desde las décadas de los sesenta y setenta han emigrado a la costa o a urbes industriales (ciudad de Alcoy) para buscar mejores alternativas laborales.

La didáctica que ofrece este centro también es apta para todas las edades e incluso para determinadas ciencias donde los aspectos climáticos son fundamentales, caso de la Geografía, Ciencias Ambientales, Turismo, etc. Sin embargo, como pusieron de manifiesto los precursores del Museo del Clima de Beniarrés, aún quedan muchas cosas por hacer e ideas didácticas por desarrollar. La importancia que pueden tener determinados centros de interpretación o como es el caso del museo que se presenta en este trabajo, puede tener sus repercusiones futuras en relación con el aprendizaje y cultura que puedan aprender los alumnos. En este sentido, 


\section{Figura V \\ Potencialidades para la enseñanza del Museo del Clima de Beniarrés}

Fuente: Elaboración propia.

\section{Potencialidades didácticas del Museo del Clima de Beniarrés}

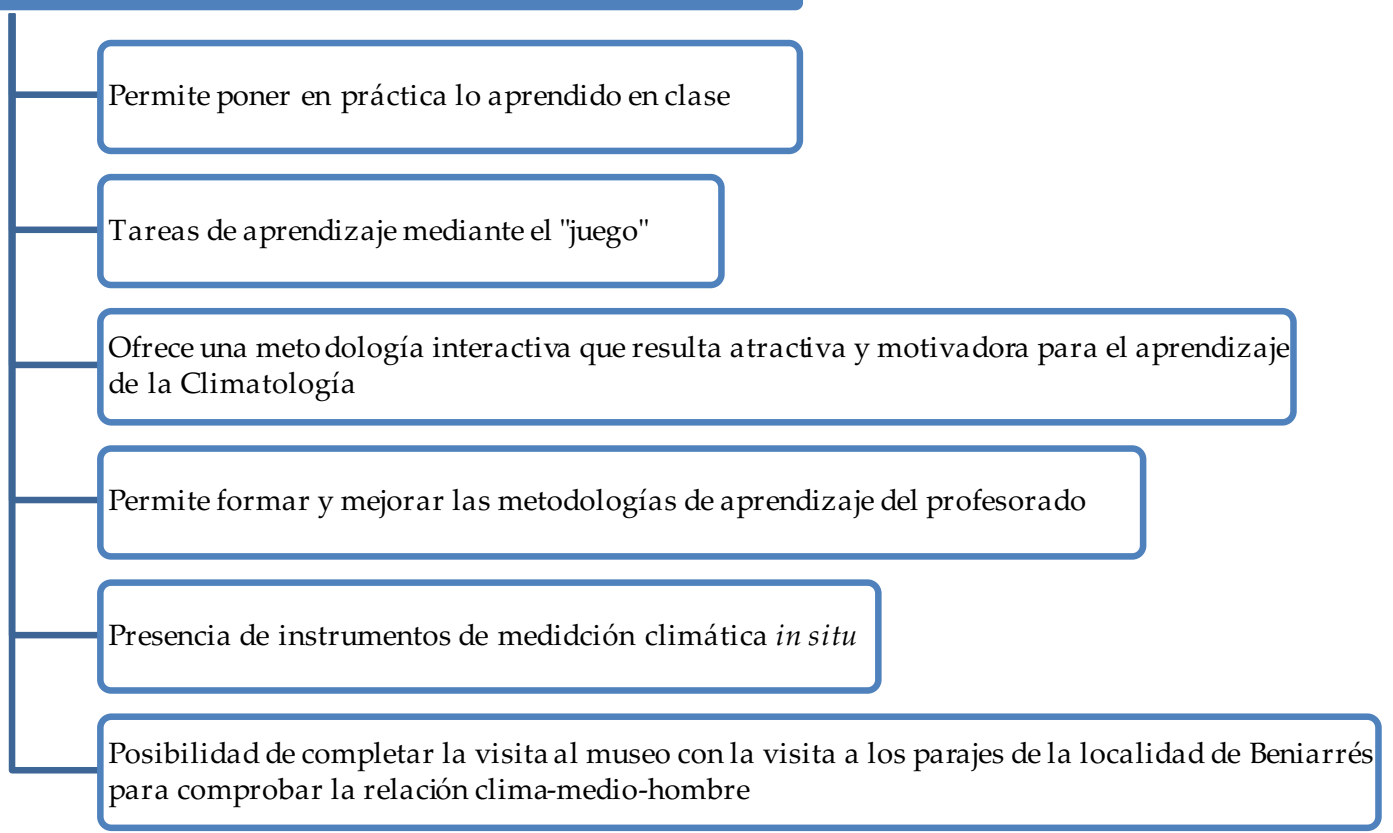

desde el ámbito educativo, también se exige una intervención de compromiso que permita trasladar a generaciones de alumnos un arma muy valiosa en su futura valoración y uso de los bienes y las experiencias patrimoniales, que como ciudadanos adultos, transmitirán a las generaciones futuras y que se convierte en una estrategia, ya que contribuye a la formación integral de los ciudadanos que transmiten vivencias y experiencias (García-Marín et al., 2015). La formación que puede adquirir el alumnado sobre el conocimiento de su medio cultural y natural, además del valor personal, sin duda colaborará en las futuras políticas relacionadas con el territorio y su gestión. La educación en el conocimiento del territorio, de la cultura de una sociedad y de su patrimonio es sumamente importante para la posterior valoración y conservación del medio, ya que los niños de ahora, serán en un futuro los que colaborarán con la creación de políticas relacionadas con la gestión del territorio y de los recursos (Martínez-Valcárcel et al., 2015).

Con este recurso didáctico se ha proporcionado un ejemplo a tener en cuenta a la hora de confeccionar el material 
y metodología docente en relación con las asignaturas que aborden conceptos sobre la Climatología, al igual que poner a disposición de la comunidad educativa alicantina una serie de itinerarios sobre los que "guiar" al profesor (para que sepa posteriormente trasmitir y enseñar a sus alumnos) en el conocimiento territorial de su espacio geográfico más próximo. Esto podría permitir a los alumnos mejorar la comprensión de los elementos geográficos del entorno más inmediato $\mathrm{y}$, por esta vía, analizar las dinámicas y relaciones socio-ambientales y culturales con el clima y para ser capaces, como futuros ciudadanos, de afrontar de manera crítica y comprometida los problemas fundamentales de nuestro mundo.

\section{Referencias bibliográficas}

BALE, J. (1996). Didáctica de la geografía en la escuela primaria. Madrid: Ministerio de Educación y Ciencia y Ediciones Morata.

BOCCONI, S., KAMPYLIS, P. y PUNIE, Y. (2012). Innovating Learning: Key Elements for Developing Creative Classrooms in Europe. Sevilla: European Commission - Joint Research Center - Institute for Prospective Technological Studies.

CORDERO, R. (2005). María Montessori y el medio ambiente como método activo. En Veinte experiencias educativas exitosas en el mundo (89-100). Nuevo León: Centro de Altos Estudios e Investigación Pedagógica.
CRESPO, J.M. (2012). Un itinerario didáctico para la interpretación de los elementos físicos de los paisajes de la Sierra de Guadarrama. Didáctica Geográfica, 13, 15-34.

DE MIGUEL, R. (2013). Geoinformación e innovación en la enseñanzaaprendizaje de la geografía: un reto pendiente en los libros de texto de secundaria. Didáctica de las Ciencias Experimentales y Sociales, $\mathrm{n}^{0} 27$, 67-90. DOI: 10.7203/DCES.27.2344

DELGADO, J. J. y RODRIGO, J. (2012). El trabajo de campo y las competencias geográficas en el estímulo para el estudio de la geografía: aplicación en un aula de $2^{\circ}$ de bachillerato. Didáctica Geográfica, nº 13, 35-56.

GARCÍA, A. L. (1994). Los itinerarios didácticos: una de las claves para la enseñanza y comprensión de la Geografía. Iber, no 1, 117-125.

GARCÍA-MARÍN, R., MARTÍNEZ-VALCÁRCEL, N., ESPEJO-MARÍN, C. y PAREDES-GUERRERO, A. (2015). Patrimonio, educación y turismo responsable: La importancia del conocimiento del patrimonio tras los estudios de bachillerato. En R. SEBASTIÁ ALCARAZ y E. TONDA MONLLOR (eds.), Investigar para innovar en la enseñanza de la Geografía (55-72). Alicante: CEE Limencop.

GÓMEZ, A. (1986). Los itinerarios pedagógicos como recurso didáctico en la enseñanza de Geografía en EGB. Didáctica Geográfica (Primera Época), no 14, 109-116. 
GONZÁLEZ, J.F. y GARCÍA, F.F. (2014). Las concepciones de los alumnos sobre el medio urbano y sus implicaciones didácticas. Un estudio en Bogotá. Didáctica Geográfica, $\mathrm{n}^{\circ}$ 15, 41-60.

MARRÓN, M. J. (2001). Geografía y Literatura. Un itinerario didáctico para la enseñanza-aprendizaje de la Geografía a partir del Lazarillo de Tormes. En M. J. MARRÓN GAITE, (Ed.), La formación geográfica de los ciudadanos en el cambio de milenio (307-335) Madrid: Asociación de geógrafos españoles. Grupo de didáctica de la Geografía.

MARRÓN, M.J. (2011). Educación geográfica y formación del profesorado. Desafíos y perspectivas en el nuevo Espacio Europeo de Educación Superior. Boletín de la Asociación de Geógrafos Españoles, n 57, 313-341.

MARTÍNEZ, N., VALLS, R. y PINEDA F. (2009). El uso del libro de texto de Historia de España en Bachillerato: diez años de estudio (1993-2003) y dos reformas (LGE-LOGSE). Didáctica de las Ciencias Experimentales y Sociales, $\mathrm{n}^{\circ}$ 23, 3-35.

MARTÍNEZ-FERNÁNDEZ, L.C., FERNÁNDEZ-VEGA, B. y MOLINA DE LA TORRE, I. (2015). Propuesta didáctica para la interpretación del espacio geográfico: La ciudad de Segovia y su entorno. Didáctica Geográfica, $\mathrm{n}^{\circ} 16,135-164$.

MARTÍNEZ-MEDINA, R. y LÓPEZFERNÁNDEZ, J.A. (2015). La enseñanza de la Climatología en los manuales escolares de ciencias sociales en educación primaria. En R. SEBASTIÁ ALCARAZ y E. TONDA MONLLOR (eds.), Investigar para innovar en la enseñanza de la Geografía (238-251). Alicante: CEE Limencop.

MARTÍNEZ-VALCÁRCEL, N., GARCÍA-MARÍN, R., ESPEJO-MARÍN, C. y MORENO-MARTÍNEZ, M.T. (2015). El aprendizaje del patrimonio: incidenia de los distintos niveles educativos, familia, asociaciones y medios de comunicación. En R. SEBASTIÁ ALCARAZ y E. TONDA MONLLOR (eds.), Investigar para innovar en la enseñanza de la Geografía (103-120). Alicante: CEE Limencop.

MÍNGUEZ, Mª . C. (2010). El paisaje como objeto de estudio de la Geografía. Un itinerario didáctico en el marco de la semana de la ciencia de la Comunidad de Madrid. Didáctica Geográfica (Segunda época), $\mathrm{n}^{\circ} 11$, 37-62.

MOROTE, A.F. (2015). El Laboratorio de Climatología de la Universidad de Alicante. Enseñanza, divulgación e investigación de la Geografía. En R. SEBASTIÁ ALCARAZ y E. TONDA MONLLOR (eds.), Investigar para innovar en la enseñanza de la Geografía (267-280). Alicante: CEE Limencop.

ORTEGA, N. (1998). El descubrimiento cultural de la Sierra de Guadarrama. En Madrid y la Sierra de Guadarrama (81-113). Madrid: Museo Municipal de Madrid. 
ROBERTS, M. (2011). What makes a geography lesson good?. Guilford: Geographical Association.

SÁNCHEZ, A. (1996). El Trabajo de campo y las Excursiones. En A. MORENO JIMÉNEZ y M. J. MARRÓN GAITE (Eds.), Enseñar Geografía, de la teoría a la práctica (160-184). Madrid: Editorial Síntesis.

SANDOYA, M.A. (2005). Estrategias didácticas para jóvenes profesores de Geografía. Didáctica Geográfica, n 7 , 535-549.

SANTANA, D., MORALES, A.J., COLOMER, J.C., CAMPO, B. y CAURÍN, C. (2015). Parques Naturales: La necesaria conceptualización transforamdora en la Educación Primaria y
Secundaria. Didáctica Geográfica, n ${ }^{\circ}$ 16, 73-94.

SOUTO, X. M. (2010). ¿Qué escuelas de Geografía para educar en ciudadanía? Didáctica de las Ciencias Experimentales y Sociales, $\mathrm{n}^{\circ}$ 24, 25-44.

TONDA, E. y SEBASTIÁ. R. (2003). Las dificultades en el aprendizaje de los conceptos de tiempo atmosférico y clima: la elaboración e interpretación de climogramas. Revista de Educación de la Universidad de Granada, $\mathrm{n}^{\mathrm{o}} 16,47-69$.

VALBUENA, M. y VALVERDE, J.A. (2006). La Climatología Local: Procedimiento para su enseñanza y aprendizaje. Didáctica Geográfica, $\mathrm{n}^{\circ} 8$, 93-108. 
